\title{
Philosophiques
}

\section{Alain Badiou, Saint Paul. La fondation de l'universalisme, Paris, PUF, 1997, 119 p.}

\section{Alain Beaulieu}

Volume 26, numéro 2, automne 1999

La critique de la raison en Europe centrale

URI : https://id.erudit.org/iderudit/004898ar

DOI : https://doi.org/10.7202/004898ar

Aller au sommaire du numéro

Éditeur(s)

Société de philosophie du Québec

ISSN

0316-2923 (imprimé)

1492-1391 (numérique)

Découvrir la revue

Citer ce compte rendu

Beaulieu, A. (1999). Compte rendu de [Alain Badiou, Saint Paul. La fondation de l'universalisme, Paris, PUF, 1997, 119 p.] Philosophiques, 26(2), 373-375.

https://doi.org/10.7202/004898ar

Ce document est protégé par la loi sur le droit d'auteur. L'utilisation des services d'Érudit (y compris la reproduction) est assujettie à sa politique d'utilisation que vous pouvez consulter en ligne.

https://apropos.erudit.org/fr/usagers/politique-dutilisation/ 


\title{
Comptes rendus
}

\author{
Alain Badiou, Saint Paul. La fondation de \\ l'universalisme. Paris, PUF, 1997, 119 p.
}

Contrairement à ce que son titre laisse penser, ce livre ne s'adresse pas en premier lieu aux amateurs d'exégèse biblique. $M$ algréles références aux textes sacrés, le Paul dont il est ici question n'est ni un apôtreni un saint. Fier de son expérience au sein de diverses organisations d'intellectuels de gauche, Badiou tente plutôt de tirer les conséquences del'hypothèse à peine fantaisiste qui fait de Paul « un Lénine dont le C hrist aurait été le $M$ arx équivoque » (p. 2). La question posée dans cet essai, plus explicite dans d'autres textes du même auteur, est la suivante : saurions-nous reconnaître les vrais résistants de notre époque, celle de la mondialisation et de l'homogénéisation identitaire? Plus radicalement : que font les révolutionnaires à l'heure de l'émergence de la pensée unique placée sous l'égide de notre régime capitalisto-démocratique dominant? Le Paul que propose d'étudier Badiou porte donc moins la soutane qu'il ne revêt l'habit du résistant à l'époque des débuts du despotisme militaire de l'empire romain. Et son époque est mutatis mutandis encore la nôtre. C'est le caractère intemporellement révolutionnaire de Paul que Badiou souhaite mettre en évidence, jusqu'à mobiliser, de façon peu orthodoxe, un vocabulaire politiquement moderne pour rendre compte del'entreprise de Paul. Celui-ci devient ainsi le chef de parti ou defaction, un activiste qui rencontre des adhérents, des sympathisants et des opposants et qui, de surcroît, doit veiller à l'organisation des collectes.

Badiou voit dans l'œuvre de Paul celle d'un grand militant en tant que ce dernier, comme dans toute grande politique, a un message à livrer. Cette annonce consiste essentiellement pour Paul, au cours des différentes épîtres, à propager sa foi dans le seul É vénement-R ésurrection. Pour élaborer sa propre théorie de l'événement, Badiou tire parti de la relativisation par Paul des messages périphériques du Christ et des miracles qui viendraient témoigner de son caractère divin. Pour Paul, l'essentiel se magnifie dans le strict Événement de la résurrection. Événement qui ne requiert nulle preuve, qui n'est absolument pas objet de connaissance et qui n'en appelle qu'à la seule conviction individuelle. Ce qui renvoie à l'une des préoccupations majeures de toute l'œuvre de Badiou, à savoir celle de l'événement indémontrable, inconnaissable et inconditionné propre à engendrer de l'impensable et de l'inimaginable. En ce sens, I'œuvre de Paul devient emblématique de la philosophie d'aujourd'hui, nous dit Badiou, non seulement parce qu'elle pose la question de la reconnaissance possible du résistant, mais aussi parce que nous vivons dans une époque où, plus que jamais, la conceptualisation trouve ses limites et où l'événement en général devient l'enjeu décisif de la pensée.

Si Paul nous est si présent, c'est parce qu'il est parvenu, par ses prédications centrées autour du seul Événement-R ésurrection, à pousser l'expérience événementielle à une certaine limite en universalisant un fait singulier donné comme commencement absolu et n'engageant que la seule conviction du croyant. C'est au nom de cette refondation événementielle à caractère universel que Paul deviendra dissident à la fois de la sagesse grecque, de la religion juive et des Chrétiens faibles (ceux, par exemple, qui se disputent autour de la question, si peu signifiante aux yeux de Paul, 


\section{4 · Philosophiques / Automne 1999}

de la circoncision). II militera au nom de la reconnaissance de cette cause impersonnelle qu'est l'Événement-R ésurrection et qui doit pouvoir générer une conviction nouvelle chez les gens, qu'ils soient juifs, grecs, femmes ou hommes de tous lieux. D'où la puissance d'universalité du message paulinien.

Ce qui fascine Badiou, ce sont les conditions d'une telle " singularité universelle» (p. 14), conditions qui seraient également celles de notre philosophie aujourd'hui. Ce qui veut dire que (si l'on excepte le contenu de la résurrection du Christ) la tâche de Paul demeure exemplairement la nôtre encore aujourd'hui : rendre compte des événements, ces inconnaissables, ultimement dans leur puissance de transformation universelle. Comme on le voit, la question de la résultante historique de la diffusion du message christique par Paul au niveau religieux est laissée de côté au profit d'un examen de l'événement dans sa puissance à générer des révolutions pour lesquelles l'œuvre de Paul devient exemplaire. L'événement singulier est toujours, pour parler comme M allarmé (l'un des principaux chevaux de bataille de Badiou avec $G$ ödel et Cantor), tel un coup de dés qui tranche dans le réel jusqu'à pouvoir prétendre à I'universalité d'une nouvelle fondation. Au cours de I'histoire, Badiou croit que certains philosophes et artistes ont su décrypter dans le coup de dés de Paul son caractère universel jusqu'à faire résonner, en écho, le style paulinien dans leurs œuvres. A u nombre de ces héritiers de la pensée événementielle de Paul, Badiou examine les cas de Pascal, qui a dû créer un discours lui permettant de concilier sa foi religieuse avec les progrès de la science, du cinéaste Pasolini, qui projetait de réaliser un film sur la vie de Paul transposée dans nos métropoles contemporaines, et de $\mathrm{N}$ ietzsche, qui se voyait lui-même comme un destin pour I'humanité, un événement en soi.

Le lecteur ne manquera pas de remarquer combien l'opacité stylistique des dernières sections tranche avec la limpidité des précédentes. $M$ ais peut être n'est-celà un obstacle que pour les nouveaux adeptes de la pensée de Badiou. Q uoi qu'il en soit, cette dernière partie rassemble huit théorèmes typiques de son style qui découlent de la pensée axiomatique paulienne de l'événement. Citons-en deux : " L'événement seul, comme contingenceillégale, fait advenir une multiplicité en excès sur elle-même et donc la possibilité d'outrepasser la finitude » (p. 85) et « Un sujet fait loi non littérale de l'adresse universelle de la vérité dont il soutient le processus » (p. 92). Pour une meilleure compréhension de ces théorèmes, il est souhaitable d'avoir en tête quelques notions spécifiques (I'Un, la finitude, la multiplicité, la procédure de vérité) thématisées dans les précédents livres de Badiou et principalement dans L'être et l'événement (Paris, Seuil, 1988).

En somme, le livreSaint-P aul. L a fondation del'universalisme constitue un formidable plaidoyer en faveur d'une pensée de l'événement. 0 n ne peut que saluer I'inventive interprétation de Paul par Badiou, qui en fait rejaillir une contemporanéité possible. Cependant, malgré le soin avec lequel Badiou souligne, dans le prologue, son caractère « héréditairement irréligieux », on se demande quand même si son livre n'est pas à placer dans une certaine mouvance interne au champ de la philosophie française depuis une dizaine d'années en faveur d'un retour à la parole sacrée, voire même à l'élaboration contagieuse d'une sorte de philosophie religieuse. En effet, on peut dire que l'époque du grand mépris post-soixante-huitard affiché à l'égard des textes religieux et fondateurs est aujourd'hui devenue, au moins en France, quelque chose du passé. O utre la dernière génération de phénoménologues ( $M$ arion, $C$ hrétien, $\mathrm{H}$ enry, disciples de Lévinas) rassemblés autour de préoccupations théologiques 
communes, on voit aussi les initiateurs de la déconstruction ( $D$ errida, de façon récurrente) et de la postmodernité (Lyotard dans son livre posthume sur saint A ugustin) se replier sur des textes religieux pour y puiser des éléments d'une philosophie présentée comme originale. La pensée de Badiou, contemplateur déclaré de la religion, est-elle à placer dans ce panorama philosophico-religieux? Badiou a-t-il arrêté son choix sur saint Paul de façon aussi désintéressée qu'il l'affirme? C'est à se demander si on en a déjà terminé avec le problème des singularités posé en termes strictement conceptuels. L'avenir nous dira si saura persister ce caractère intempestif, jusqu'à maintenant triomphant dans les écrits « antiphilosophiques » de Badiou, qui demeure quand même aujourd'hui le penseur le plus original en France.

ALAIN BEAULIEU

Université de Paris VIII Vincennes-St-Denis 